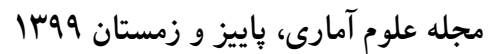

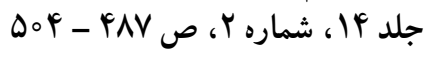

DOI: $10.29252 /$ jss.14.2.487

\section{تحليل دادههاى طول عمر وايبول تحت تعمير ناقص}

$$
\begin{aligned}
& \text { هدى كامرانفر، جواد اطمينان و مجيد جهركندى } \\
& \text { كروه آمار، دانشكاه بيرجند }
\end{aligned}
$$

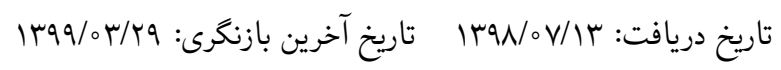

جكيده: سيستم تعميريذيرى با دو نوع خرابى مورد مطالعه قرار كرفته است. خرابى نوع يك با با تعمير

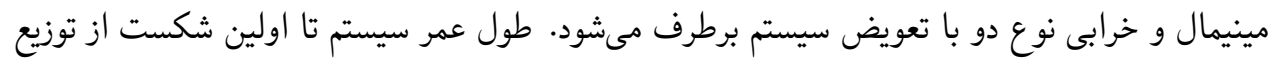

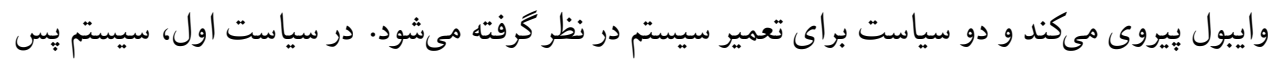

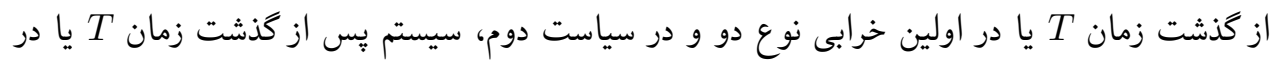

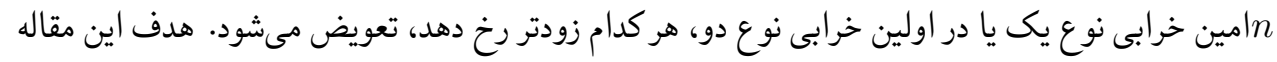

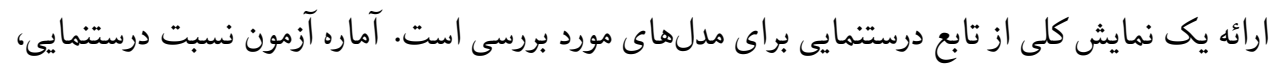

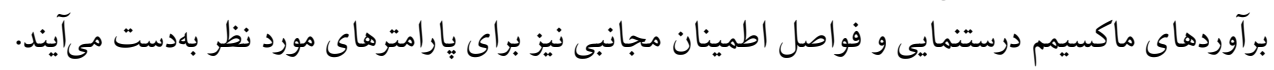

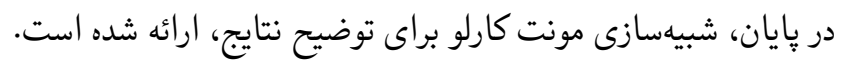

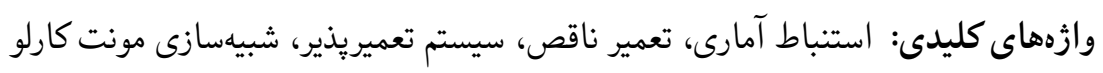

\section{|}

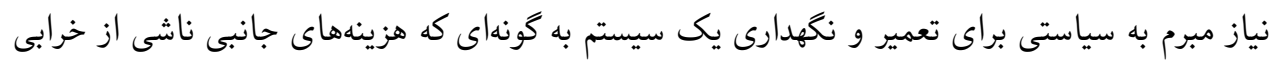

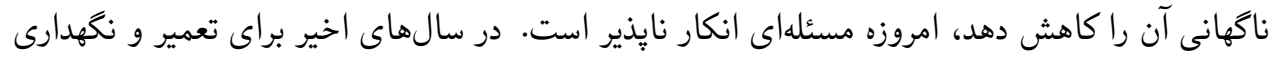

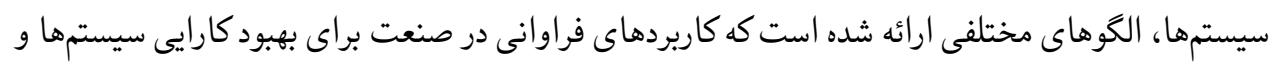


دادههاى طول عمر تحت تعمير ناقص

تجهيزات دارند. در مدلهاى اوليه مطرح شده، فرايندهاى سادهاى براى توصيف رفتار سيستمهاى تعميريذير

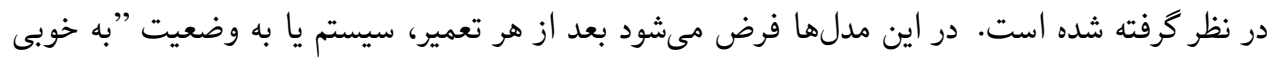

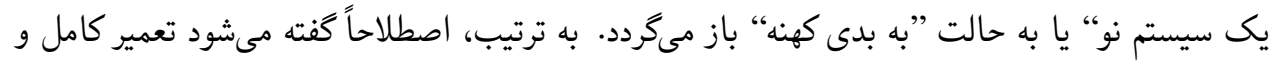

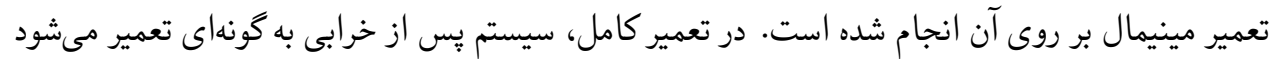

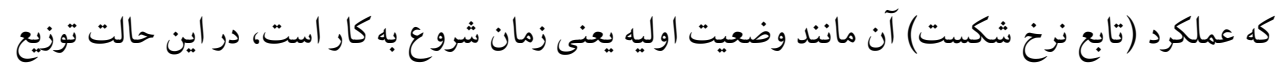

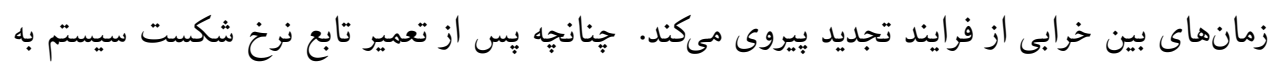

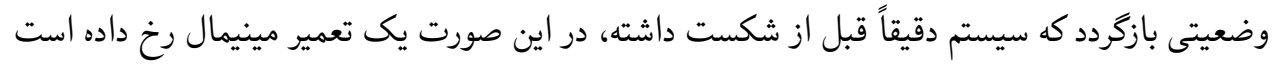

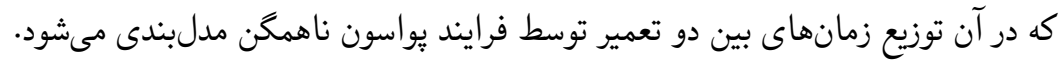

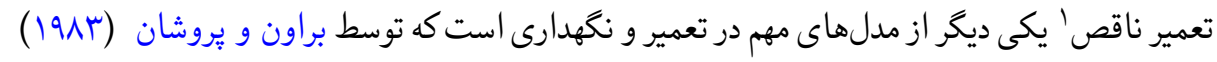

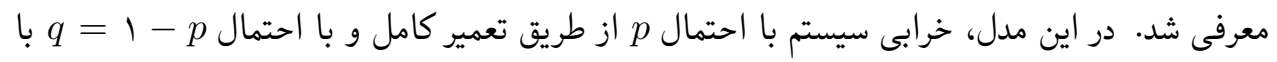

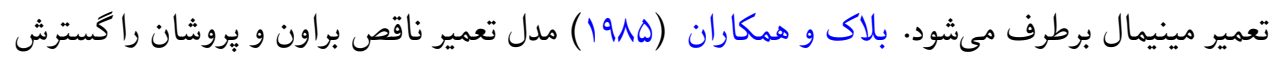

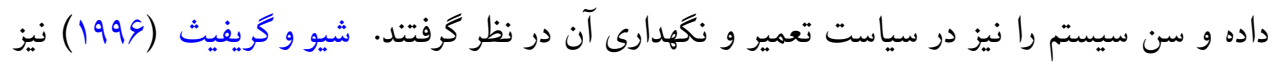

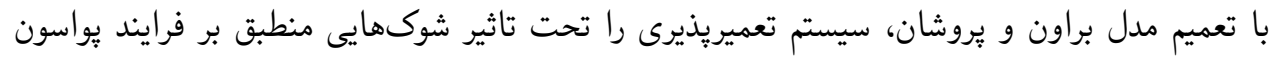

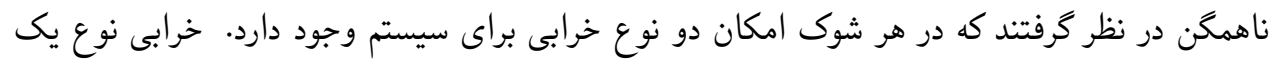

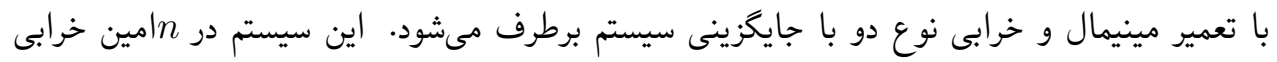

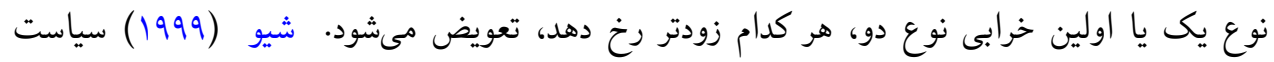

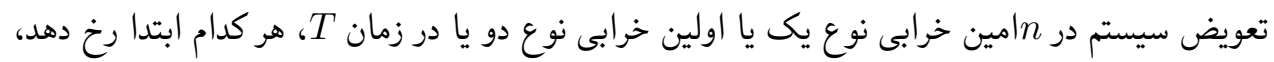

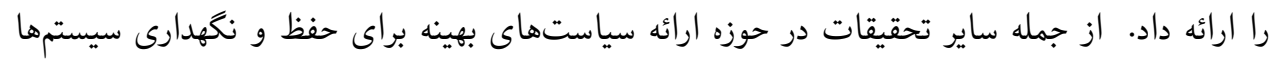

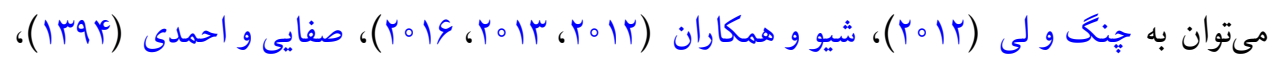

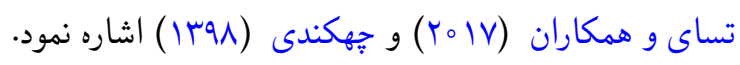
در تحقيقات حوزه قابليت اعتماد، اغلب سيستهها از نقطه نظر مهندسى مورد بررسى قرار مىگيرند.

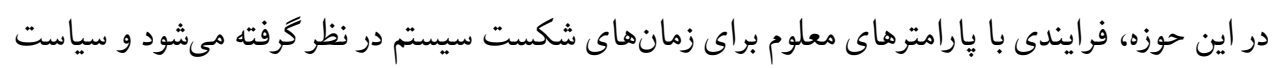

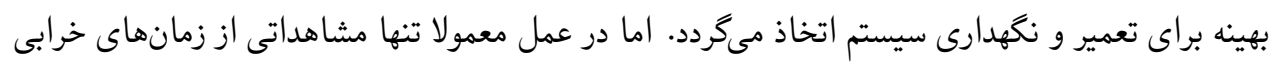

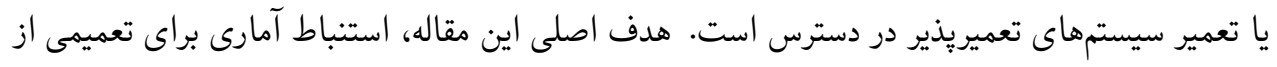

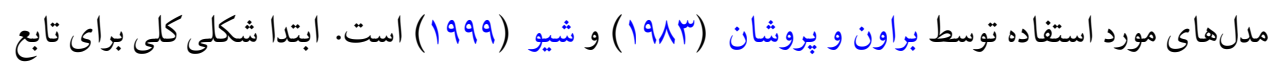

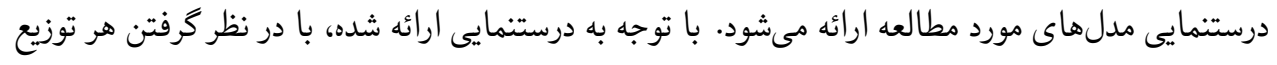


طول عمر اوليه دلخواه براى سيستم تعميريذير مىتوان به استنباط آمارى درباره پارامترهاى مدل يرداخت و

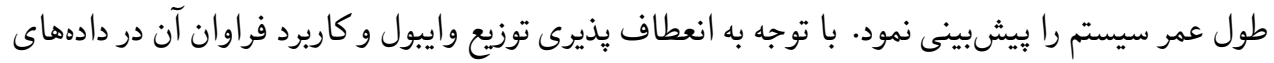

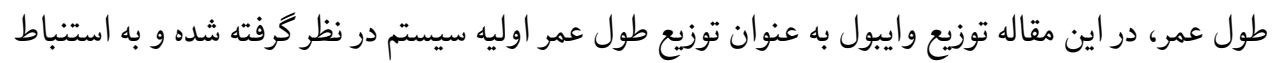

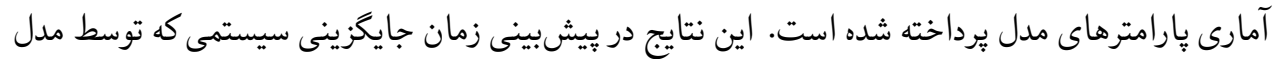

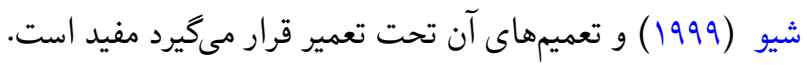

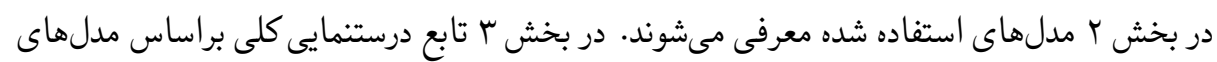

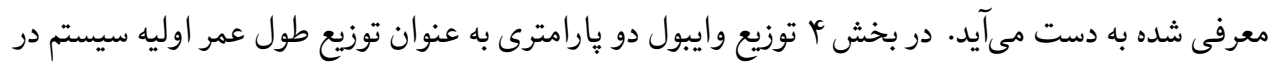

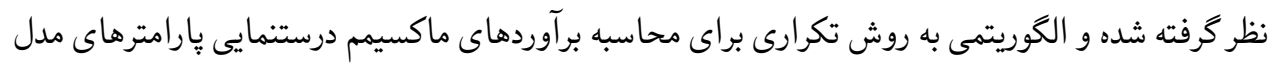

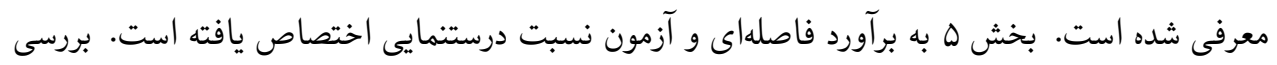

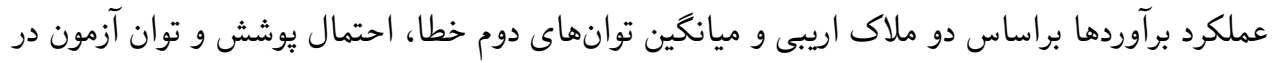

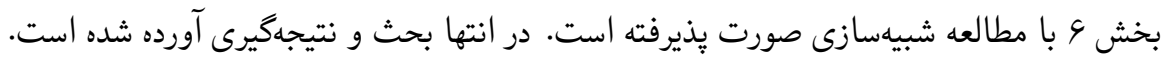

\section{ب}

سيستم تعميريذيرى را در نظر بخيريد كه از زمان صفر شروع به كار مىكند و تحت تاثير شوكهايى منطبق

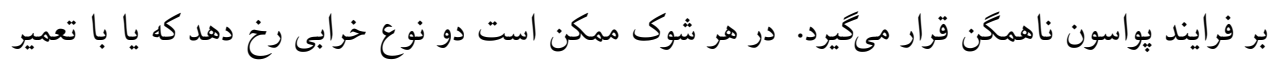

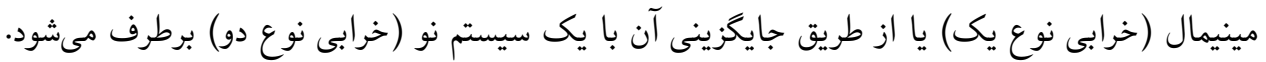

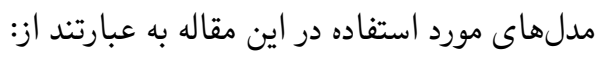

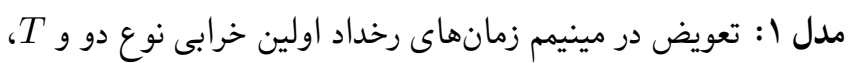

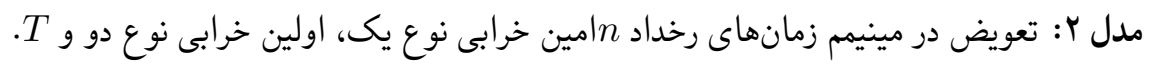

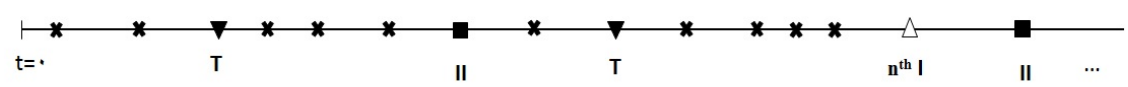

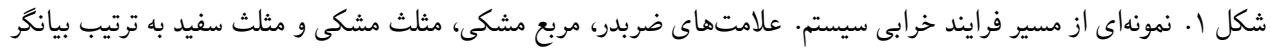

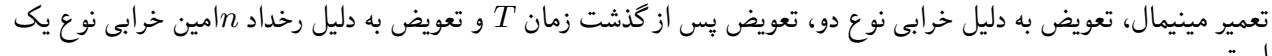

با توجه به اينكه مدل Y جامعتر است، در شكل ا نمونهاى از خرابىهاى متوالى تحت اين مدل نمايش داده شده است. فرض كنيد $)$

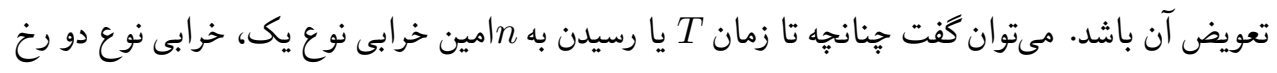


ندهد با نوعى سانسور مواجه هستيم. قابل ذكر است كه فرض شده سيستم به كونهاى طراحى شده است كه

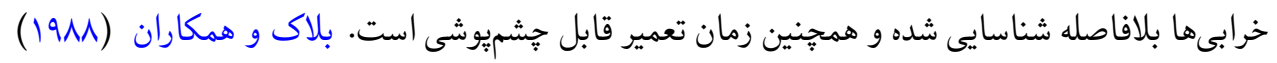

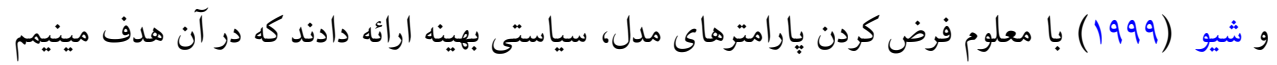

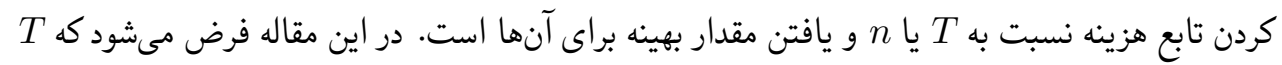

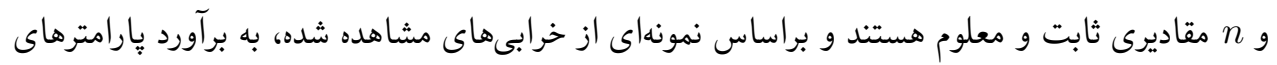
قابليت اعتماد و اثر تعمير برداخته خواهد شد.

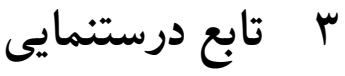

مدل ا: ابتدا تابع درستنمايى براى هر يك از حالتهاى رخداد خرابى نوع دو قبل و بعد از زمان سميس تابع درستنمايى براى حالت كلى ارائه مىشود. ا. خرابى نوع دو قبل از زمان T رنخ داده است. در اين صورت

$$
\begin{aligned}
L(p, F \mid \boldsymbol{x}, m) & =q^{m-1} p \prod_{i=1}^{m} f\left(x_{i} \mid x_{i-1}\right) \\
& =q^{m-1} p \prod_{i=1}^{m} \frac{f\left(x_{i}\right)}{\bar{F}\left(x_{i-1}\right)} \quad \circ=x_{\circ}<x_{1}<\ldots<x_{m}, \\
& =q^{m-1} p \bar{F}\left(x_{m}\right) \prod_{i=1}^{m} \frac{f\left(x_{i}\right)}{\bar{F}\left(x_{i}\right)}
\end{aligned}
$$

كه در آن تابع بقا طول عمر اوليه سيستم است.

r. ت تا زمان T خرابى نوع دو رخخ نداده است. در اين حالت

$$
L(p, F \mid \boldsymbol{x}, m)=q^{m} \bar{F}(T) \prod_{i=1}^{m} \frac{f\left(x_{i}\right)}{\bar{F}\left(x_{i}\right)}, \quad \circ<x_{1}<\ldots<x_{m},
$$




$$
\begin{aligned}
\text { با تعميم دو حالت فوق، تابع درستنمايى در حالت كلى به صورت }=q^{m} \bar{F}(T)\left(\frac{p}{q} \frac{\bar{F}\left(x_{m}\right)}{\bar{F}(T)}\right)^{I(z=1)} \prod_{i=1}^{m} \frac{f\left(x_{i}\right)}{\bar{F}\left(x_{i}\right)},
\end{aligned}
$$

است، كه در آن (I) I I I تابع نشانكر رخداد خرابى نوع دو قبل از زمان T است. بنابراين تابع

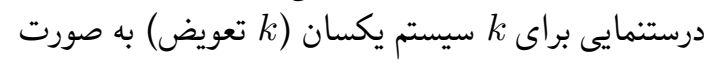

$$
L(p, F \mid \tilde{\boldsymbol{x}}, \mathbf{m})=q^{M-N_{\uparrow}} p^{N_{\uparrow}}(\bar{F}(T))^{k-N_{\uparrow}} \prod_{j=1}^{k}\left(\prod_{i=1}^{m_{j}} \frac{f\left(x_{j, i}\right)}{\bar{F}\left(x_{j, i}\right)}\right)\left(\bar{F}\left(x_{j, m_{j}}\right)\right)^{I\left(z_{j}=1\right)}
$$

$N_{1}=M=\sum_{j=1}^{k} m_{j} ، \boldsymbol{x}_{j}=\left(x_{j, 1}, \ldots, x_{j, m_{j}}\right) ، \tilde{\boldsymbol{x}}=\left(\boldsymbol{x}_{1}, \ldots, \boldsymbol{x}_{k}\right)$ (است، كه در آن مدل r: سيستم مورد مطالعه در زمان T)

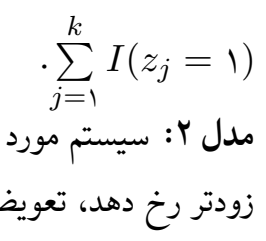

$$
\begin{aligned}
& L(p, F \mid \boldsymbol{x}, m)=q^{m-1}\left(\prod_{i=1}^{m} \frac{f\left(x_{i}\right)}{\bar{F}\left(x_{i}\right)}\right)\left(\bar{F}\left(x_{m}\right)\right)^{1-I(m<n) I(z=\circ)} p^{I(m<n) I(z=1)} \\
& \times \quad(q \bar{F}(T))^{I(m<n) I(z=\circ)},
\end{aligned}
$$

$$
L(p, F \mid \tilde{\boldsymbol{x}}, \mathbf{m})=q^{M-k+L-N_{\curlyvee}} p^{N_{\curlyvee}}(\bar{F}(T))^{L-N_{\uparrow}} \prod_{j=1}^{k}\left(\prod_{i=1}^{m_{j}} \frac{f\left(x_{j, i}\right)}{\bar{F}\left(x_{j, i}\right)}\right)\left(\bar{F}\left(x_{j, m_{j}}\right)\right)^{I_{j}}
$$

$$
\begin{aligned}
& \text { كه درآن } \\
& \text { و }
\end{aligned}
$$




\section{ب برآورد بارامترهاى مدل تحت توزيع طول عمر وايبول}

با توجه به (1) و (Y) برآورد پارامتر p مستقل از توزيع زمانهاى خرابى است. با مشتقيرى از لكاريتم

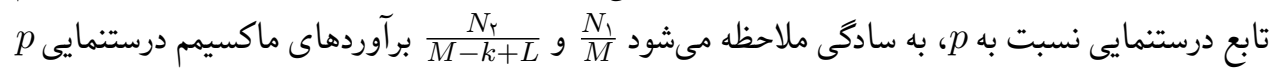

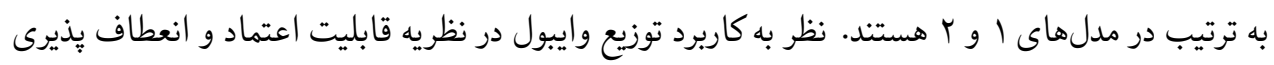

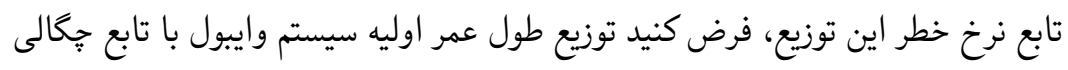

$$
f(t)=\alpha \lambda t^{\alpha-1} e^{-\lambda t^{\alpha}}, t>\circ, \alpha>\circ, \lambda>\circ
$$

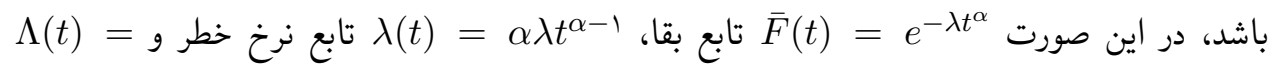

$$
\begin{aligned}
& \text { تابع نرخ خطر تجمعى هستند } \int_{0}^{t} \lambda(u) d u=\lambda t^{\alpha} \\
& \ell(\lambda, \alpha)=\log L(p, F \mid \tilde{\boldsymbol{x}}, \mathbf{m}) \\
& =\left(M-N_{\uparrow}\right) \log (q)+N_{\uparrow} \log (p)+M(\log (\alpha \lambda))-\left(k-N_{\uparrow}\right) \lambda T^{\alpha} \\
& +(\alpha-1) \sum_{j=1}^{k} \sum_{i=1}^{m_{j}} \log \left(x_{j, i}\right)-\sum_{j=1}^{k} \lambda x_{j, m_{j}}^{\alpha} I\left(z_{j}=1\right) \text {. }
\end{aligned}
$$

است، كه با مشتقكيرى از آن نسبت به $\lambda$ و $\alpha$ برآوردهاى ماكسيم درستنمايى

$$
\hat{\lambda}=\frac{M}{\left(k-N_{\curlywedge}\right) T^{\alpha}+\sum_{j=1}^{k} x_{j, m_{j}}^{\alpha} I\left(z_{j}=1\right)},
$$

$$
\hat{\alpha}=\frac{M}{\left(k-N_{\uparrow}\right) \lambda T^{\alpha} \log (T)-\sum_{j=1}^{k} \sum_{i=1}^{m_{j}} \log \left(x_{j, i}\right)+\lambda \sum_{j=1}^{k} x_{j, m_{j}}^{\alpha} \log \left(x_{j, m_{j}}\right) I\left(z_{j}=1\right)} .
$$

حاصل مىشوند. از آنجاكه (ه) نسبت به ه غيرخطى است، معمولاً براى حل اين معادله مارها از دستورهاى

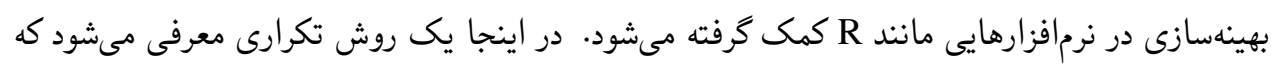

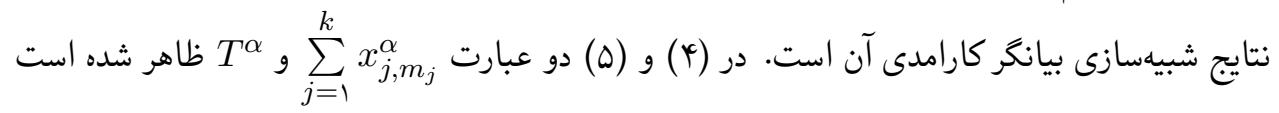


كه در ارتباط با نمونهايى هستند كه به ترتيب در آنها خرابى نوع دو (1)

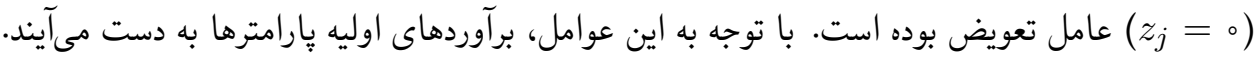

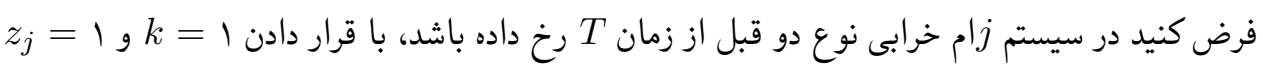

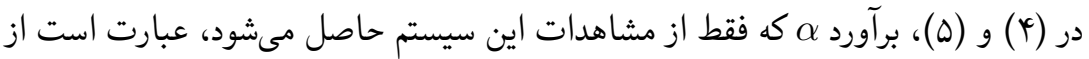

$$
\hat{\alpha}_{\circ j}=\frac{m_{j}}{m_{j} \log \left(x_{j, m_{j}}\right)-\sum_{i=1}^{m_{j}} \log \left(x_{j, i}\right)} .
$$

از طرف ديكر برآورد يارامترها بر اساس مشاهدات نمونههايى كه در آنها خرابى نوع دو قبل از زمان T رخخ

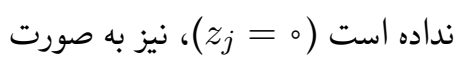

$$
\begin{aligned}
& \hat{\lambda}_{\circ}^{*}=\frac{\sum_{j=1}^{k} I\left(z_{j}=\circ\right) m_{j}}{\left(k-N_{\uparrow}\right) T^{\alpha}}=\frac{M_{\curlywedge}^{*}}{\left(k-N_{\uparrow}\right) T^{\alpha}}, \\
& \hat{\alpha}_{\circ}^{*}=\frac{M_{\uparrow}^{*}}{M_{\uparrow}^{*} \log (T)-\sum_{j=1}^{k} \sum_{i=1}^{m_{j}} \log \left(x_{j, i}\right) I\left(z_{j}=\circ\right)} . \\
& \text { بهدست مىآيد. حال مىتوان مقادير اوليه پارامترها را به صورت }
\end{aligned}
$$

$$
\hat{\lambda}_{\circ}=\frac{M}{\left(k-N_{1}\right) T^{\hat{\alpha}_{\circ}^{*}}+\sum_{j=1}^{k} x_{j, m_{j}}^{\hat{\alpha}_{o}} I\left(z_{j}=1\right)},
$$

$\hat{\alpha}_{\circ}=\frac{M}{\left(k-N_{1}\right) \hat{\lambda}_{\circ} T^{\hat{\alpha}_{\circ}^{*}} \log (T)-\sum_{j=1}^{k} \sum_{i=1}^{m_{j}} \log \left(x_{j, i}\right)+\hat{\lambda}_{\circ} \sum_{j=1}^{k} x_{j, m_{j}}^{\hat{\alpha}_{\alpha_{j}}} \log \left(x_{j, m_{j}}\right) I\left(z_{j}=1\right)}$

در نظر گرفته و براى برآورد بارامترهاى مدل ا، الخوريتمى ارائه نمود. 


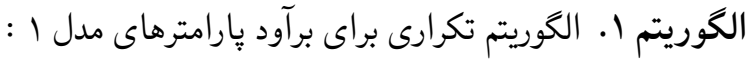

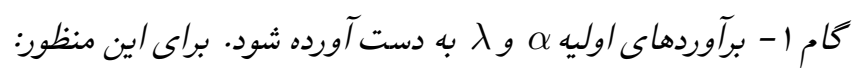

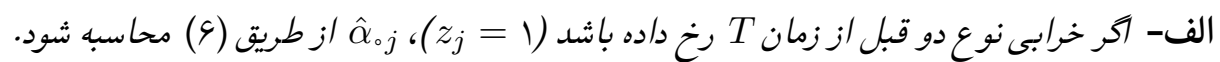

$$
\begin{aligned}
& \text { ب- آكر خرابى نوع دو قبل از زمان T رنخ نداده باشد (o } \\
& \text { ج- از طريق (^) و (9)، مقادير /وليه } \\
& \text { كام r - در مرحله lام برآورد م ور به صورت زير به روز شوند. } \\
& \hat{\lambda}_{l}=\frac{M}{\left(k-N_{1}\right) T^{\hat{\alpha}_{l-1}}+\sum_{j=1}^{k} x_{j, m_{j}}^{\hat{\alpha}_{l-1}} I\left(z_{j}=1\right)} \\
& \hat{\alpha}_{l}=\frac{M}{\left(k-N_{1}\right) \hat{\lambda}_{l} T^{\hat{\alpha}_{l-l}} \log (T)-\sum_{j=1}^{k} \sum_{i=1}^{m_{j}} \log \left(x_{j, i}\right)+\hat{\lambda}_{l} \sum_{j=1}^{k} x_{j, m_{j}}^{\hat{\alpha}_{l-1}} \log \left(x_{j, m_{j}}\right) I\left(z_{j}=1\right)} .
\end{aligned}
$$

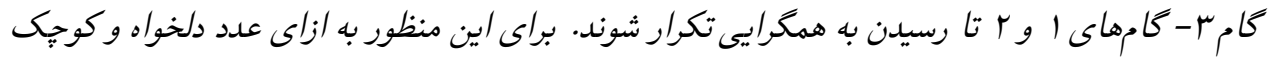

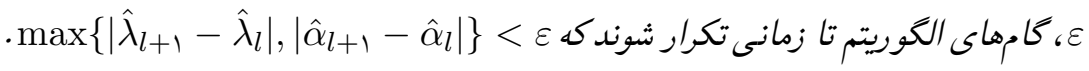

$$
\begin{aligned}
& \text { مدل Y: لكاريتم تابع درستنمايى مدل Y براساس (Y) و توزيع وايبول به صورت } \\
& \ell(\lambda, \alpha)=\left(M-k+L-N_{\Upsilon}\right) \log (q)+N_{\Upsilon} \log (p)+M \log (\alpha \lambda) \\
& +(\alpha-1) \sum_{j=1}^{k} \sum_{i=1}^{m_{j}} \log \left(x_{j, i}\right)-\left(L-N_{\uparrow}\right) \lambda T^{\alpha}-\sum_{j=1}^{k} I_{j} \lambda x_{j, m_{j}}^{\alpha} \\
& \text { است، كه با كرفتن مشتق از آن نسبت به } \alpha \text { و ג، نتيجه مىشود: } \\
& \hat{\lambda}=\frac{M}{\left(L-N_{\uparrow}\right) T^{\alpha}+\sum_{j=1}^{k} I_{j} x_{j, m_{j}}^{\alpha}}, \\
& \hat{\alpha}=\frac{M}{\left(L-N_{\uparrow}\right) \hat{\lambda} T^{\alpha} \log (T)+\hat{\lambda} \sum_{j=1}^{k} I_{j} x_{j, m_{j}}^{\alpha} \log \left(x_{j, m_{j}}\right)-\sum_{j=1}^{k} \sum_{i=1}^{m_{j}} \log \left(x_{j, i}\right)} .
\end{aligned}
$$


همانطور كه ملاحظه مىشود (11) نسبت به $\alpha$ غيرخطى است. بنابراين مشابه مدل ا، از روشى تكرارى

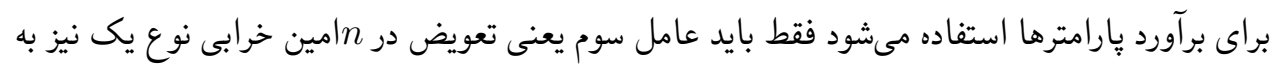

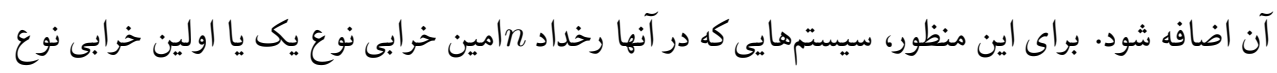

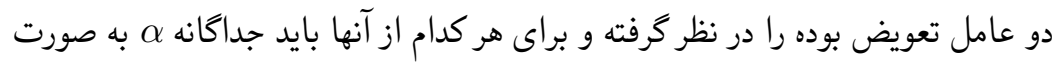

$$
\hat{\alpha}_{\circ j}=\frac{m_{j}}{m_{j} \log \left(x_{j, m_{j}}\right)-\sum_{i=1}^{m_{j}} \log \left(x_{j, i}\right)} I_{j}, j=1, \ldots, k,
$$

برآورد شود. حال بر اساس سيستهايى كه رسيدن به زمان T عامل تعويض بوده است،

$$
\hat{\lambda}=\frac{M_{r}^{*}}{\left(L-N_{\Upsilon}\right) \lambda T^{\alpha}},
$$

نتيجه مىشود، كه در آن

$$
\frac{\partial \ell(\lambda, \alpha)}{\partial \alpha}=\frac{M_{\digamma}^{*}}{\alpha}-\left(L-N_{\digamma}\right) \lambda T^{\alpha} \log (T)+\sum_{j=1}^{k} \sum_{i=1}^{m_{j}}\left(1-I_{j}\right) \log \left(x_{j, i}\right)=\circ,
$$$$
\text { مقدار اوليه برآورد م بر اساس اين سيسترها به صورت }
$$

$$
\hat{\alpha}_{\circ}^{*}=\frac{M_{r}^{*}}{M_{\uparrow}^{*} \log (T)-\sum_{j=1}^{k} \sum_{i=1}^{m_{j}}\left(1-I_{j}\right) \log \left(x_{j, i}\right)} .
$$

به دست مىآيد. با مقادير اوليه به دست آمده براى بارامترها، ادامه كار دقيقا مشابه الكوريتم ا است.

\section{ه آزمون فرضيه و فاصله اطمينان}

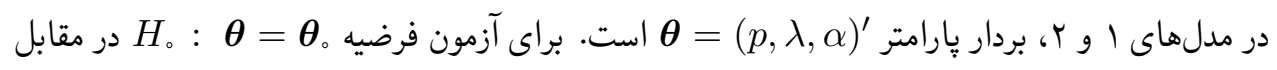

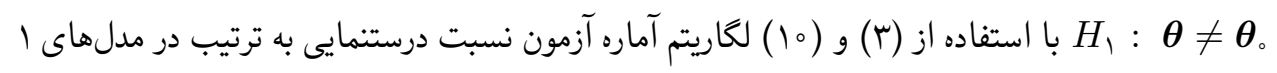




$$
\begin{aligned}
T_{\mathrm{LR}_{1}} & =M\left(\log \left(\frac{q_{\circ}}{\hat{q}}\right)+1\right)+N_{1} \log \left(\frac{p_{\circ} \hat{q}}{q_{\circ} \hat{p}}\right)+M \log \left(\frac{\alpha_{\circ} \lambda_{\circ}}{\hat{\alpha} \hat{\lambda}}\right) \\
& +\left(\alpha_{\circ}-\hat{\alpha}\right) \sum_{j=1}^{k} \sum_{i=1}^{m_{j}} \log \left(x_{j, i}\right)-\lambda_{\circ}\left[\left(k-N_{1}\right) T^{\alpha_{\circ}}+\sum_{j=1}^{k} x_{j, m_{j}}^{\alpha_{\circ}} I\left(z_{j}=1\right)\right], \\
T_{\mathrm{LR}_{\Upsilon}} & =M\left(\log \left(\frac{q_{\circ}}{\hat{q}}\right)+1\right)-N_{\uparrow} \log \left(\frac{q_{\circ} \hat{p}}{p_{\circ} \hat{q}}\right)+(L-k) \log \left(\frac{q_{\circ}}{\hat{q}}\right)+M \log \left(\frac{\alpha_{\circ} \lambda_{\circ}}{\hat{\alpha} \hat{\lambda}}\right) \\
& +\left(\alpha_{\circ}-\hat{\alpha}\right) \sum_{j=1}^{k} \sum_{i=1}^{m_{j}} \log \left(x_{j, i}\right)-\lambda_{\circ}\left[\left(L-N_{\uparrow}\right) T^{\alpha_{\circ}}+\sum_{j=1}^{k} I_{j} x_{j, m_{j}}^{\alpha_{\circ}}\right]
\end{aligned}
$$

است. آزمون فرضيه p مىتواند در بررسى مينيمال يا كامل بودن تعمير انجام شده راهگشا باشد. فواصل

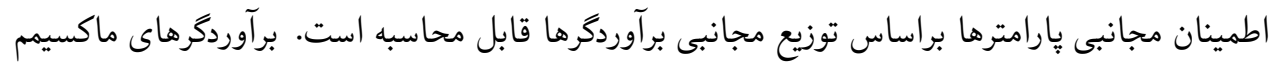

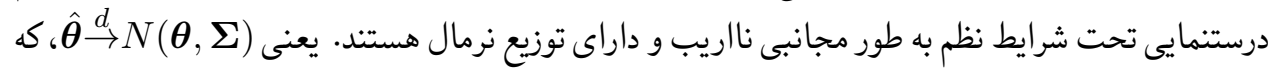

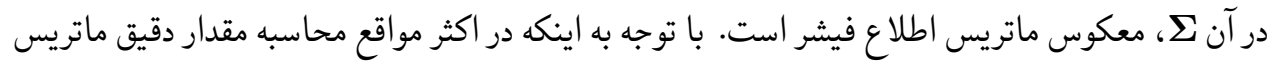
اطلاع فيشر امكان يذير نيست، از برآورد آن، يعنى إنى

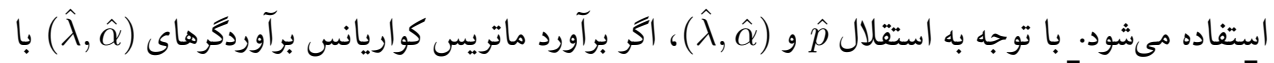

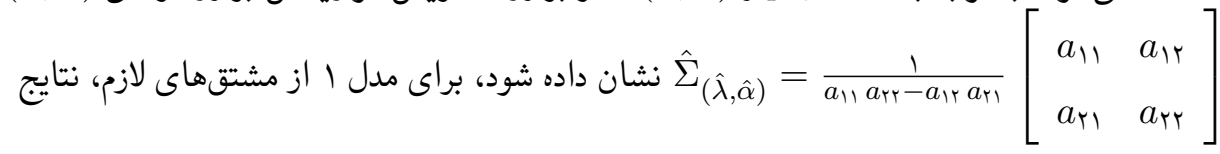

$$
\begin{aligned}
\widehat{V}(\hat{p}) & =\left.\left(-\frac{\partial^{\Upsilon} \ell}{\partial p^{\Upsilon}}\right)^{-1}\right|_{p=\hat{p}}=\frac{\hat{p}(1-\hat{p})}{M-\Upsilon N_{\uparrow}}, \\
a_{\uparrow} & =\frac{M}{\hat{\alpha}^{\Upsilon}}+\left(k-N_{\uparrow}\right) \hat{\lambda} T^{\hat{\alpha}}(\log (T))^{r}+\hat{\lambda} \sum_{j=1}^{k} x_{j, m_{j}}^{\hat{\alpha}}\left(\log \left(x_{j, m_{j}}\right)\right)^{\Upsilon} I\left(z_{j}=1\right), \\
a_{\mid \Upsilon} & =a_{\Upsilon \uparrow}=-\left(k-N_{\uparrow}\right) T^{\hat{\alpha}} \log (T)-\sum_{j=1}^{k} x_{j, m_{j}}^{\hat{\alpha}} \log \left(x_{j, m_{j}}\right) I\left(z_{j}=1\right), \\
a_{\Upsilon \Upsilon} & =\frac{M}{\hat{\lambda}^{r}}
\end{aligned}
$$




$$
\begin{aligned}
& \text { حاصل مىشوند. به طور مشابه براى مدل r نيز داريم } \\
& \widehat{V}(\hat{p})=\left.\left(-\frac{\partial^{r} \ell}{\partial p^{r}}\right)^{-1}\right|_{p=\hat{p}}=\frac{\hat{p}(1-\hat{p})}{M-k+L-r N_{\Upsilon}}, \\
& a_{11}=\frac{M}{\hat{\alpha}^{\Upsilon}}+\left(L-N_{\uparrow}\right) \hat{\lambda} T^{\hat{\alpha}}(\log (T))^{\Upsilon}+\hat{\lambda} \sum_{j=1}^{k} I_{j} x_{j, m_{j}}^{\hat{\alpha}}\left(\log \left(x_{j, m_{j}}\right)\right)^{\Upsilon} \text {, } \\
& a_{\mid \uparrow}=a_{\Upsilon \uparrow}=-\left(L-N_{\uparrow}\right) T^{\hat{\alpha}} \log (T)-\sum_{j=1}^{k} I_{j} x_{j, m_{j}}^{\hat{\alpha}} \log \left(x_{j, m_{j}}\right), \\
& a_{r r}=\frac{M}{\hat{\lambda}^{r}} \\
& \text { اكنون مىتوان فواصل اطمينان تقريبى \% }
\end{aligned}
$$

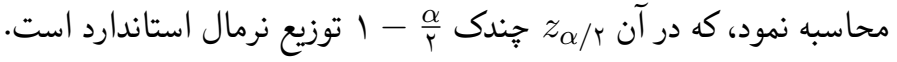

\section{9}

براى بررسى نتايج نظرى، با در نظر گرفتن (r,

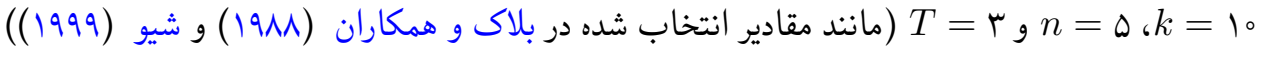

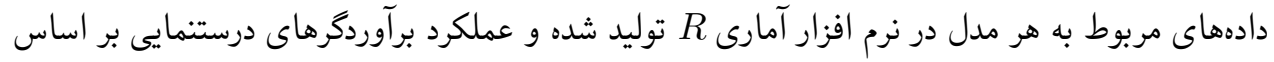

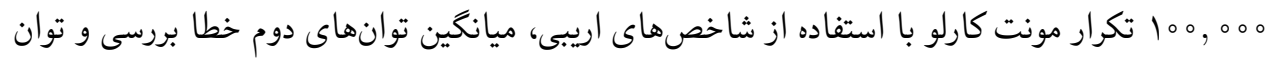
آماره آزمون نسبت درستنمايى و درصد يوشش فواصل اطمينان مجانبى برآورد مىشئ دود.

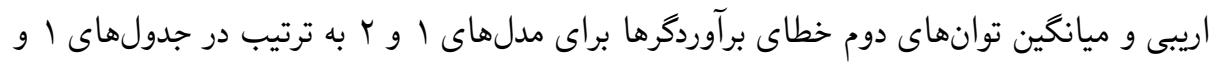

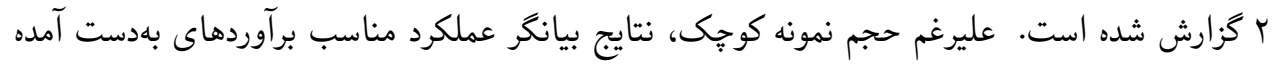

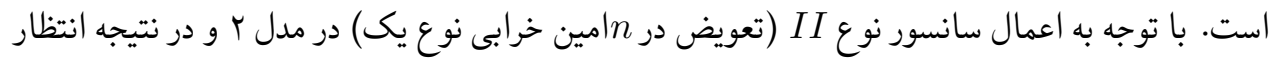

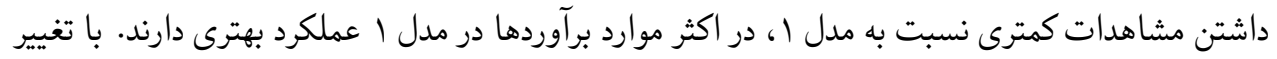

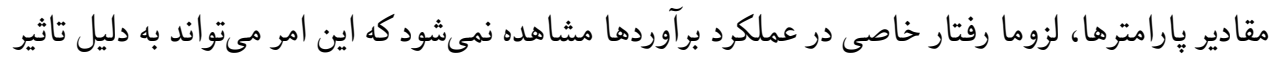

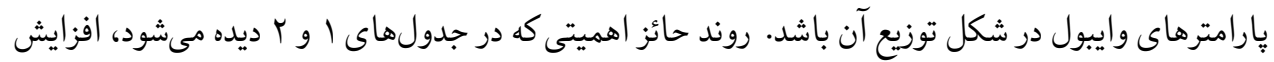

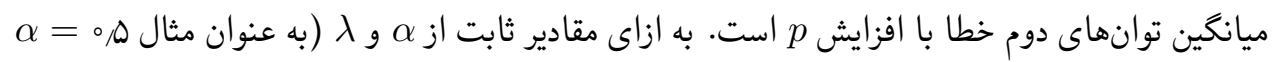

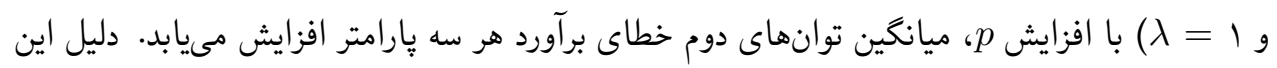




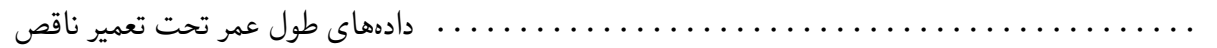

491

جدول ا. اريبى و ميانگين توانهاى دوم خطاى بارامترهاى مدل ا.

\begin{tabular}{|c|c|c|c|c|c|c|c|c|}
\hline \multicolumn{3}{|c|}{ ميانگين توانهاى دوم خطا } & \multicolumn{3}{|c|}{ اريبى } & \multirow{2}{*}{$\lambda$} & \multirow{2}{*}{$\alpha$} & \multirow{2}{*}{$\mathrm{p}$} \\
\hline$\hat{p}$ & $\hat{\lambda}$ & $\hat{\alpha}$ & $\hat{p}$ & $\hat{\lambda}$ & $\hat{\alpha}$ & & & \\
\hline$\circ, 0 \circ \Delta T$ & $0 / 1 \mathrm{VA}$ & $\circ, 0 \mid V D$ & $0,0 \circ \mu q$ & $0, \mu \backslash 14_{0}$ & זسM 0,0 & 1 & & \\
\hline$\circ, 0 \circ \mu \mathrm{F}$ & $0,19 Y V$ & 0,0094 & $\circ, 0 \circ \Delta r$ & $0,09 \circ 0$ & $0,0 \mathrm{Mr}$ & $r$ & $\circ / \Delta$ & \\
\hline $0,0 \circ Y G$ & $0, K \Delta 9 V$ & $0,009 \mu$ & $\circ, 0 \circ \Delta r$ & 0,0179 & $0,01 T V$ & $r$ & & \\
\hline$\circ, 0 \circ \mathrm{H}$ & $0,0 V 99$ & 0,0 YYq & $\circ, 0 \circ \Delta r$ & 0,0 KTD & 0,0 frq & 1 & & \\
\hline $0,0 \circ Y Y$ & $\circ / \Delta \wedge G$ & op YYO & 0,0009 & $-0,0 \circ \Delta r$ & O,OYYT & $r$ & 1 & $0 / 1$ \\
\hline 0,0019 & $\circ, \gamma \Delta 1 \circ$ & $\circ \circ \mid \Delta \Delta$ & 0,0094 & $-0,0 \circ \Delta V$ & 0,0194 & $r$ & & \\
\hline 0,0019 & 0,0991 & 0,0910 & 0,0091 & $0,000 \wedge$ & 0,0 MYY & 1 & & \\
\hline 0,0010 & $\circ / \Delta \mu_{0}$ & 0,0 prt & $0,0 \circ V G$ & $-0,000 Y$ & 0,0 TVG & $r$ & r & \\
\hline $0,0 \circ \mid \mathrm{F}$ & $0, Y 901$ & 0,0999 & $\circ, 0 \circ \wedge \Lambda$ & 0,0019 & 0,0 MYT & $r$ & & \\
\hline $0 / 0100$ & ostrV & 0,0194 & $0,0 \circ \mathrm{VG}$ & $0, \mu Y \circ \mu$ & 0,0 FYT & 1 & & \\
\hline$\circ, 0 \circ V \mu$ & 0,1191 & $0 / 0100$ & 0,0104 & $\circ / 0 \wedge Y D$ & 0,0 YTV & $r$ & $\circ / 0$ & \\
\hline 0,0090 & oxtrVo & $\circ, \circ \circ V D$ & 0,0111 & ० YイAG & 0,0190 & $r$ & & \\
\hline$\circ, 0 \circ \mathrm{VA}$ & $0 / 0 \wedge T Y$ & 0,0 Y $\Lambda_{\circ}$ & $0,009 \pi$ & $0,001 Y$ & ه & 1 & & \\
\hline$\circ, \circ \circ \Delta V$ & $\circ / \mathrm{VAV}$ & 0,0 TVT & $0,011 \mathrm{~V}$ & $0,0 \circ \wedge \hat{F}$ & $0,0 \mu_{0}$ & $r$ & 1 & 11 \\
\hline$\circ / \circ \circ \Delta \circ$ & $\circ, \mu \circ q$ & 0,0 YT. & $0,0|M|$ & $0,0 Y \circ Y$ & $0,0 Y \Lambda I$ & $r$ & & \\
\hline$\circ / \circ \circ \downarrow \circ$ & $0 / 0 V G Y$ & 0,0900 & $0,0 \mid H Y$ & 0,0001 & $\circ, 0 \Delta \Delta T$ & 1 & & \\
\hline $0,0 \circ 49$ & $0, \pi \circ 01$ & 0,1909 & 0,0109 & $-0,0010$ & $0,0 \mathrm{VDI}$ & r & r & \\
\hline$\circ, 0 \circ 4 \Delta$ & 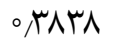 & $\circ, x \circ V$ & $0,0 \mid V_{0}$ & 0,0 YOI & $0 / 11 V_{0}$ & $r$ & & \\
\hline $0,0|f|$ & $0,194 T$ & 0,0 TOA & $0,010 \%$ & سMVTR & $\circ, 0019$ & 1 & & \\
\hline 0,0110 & & سTוס0 & $0,0 \mid \mathrm{kT}$ & $0 / Y I V$ & $0,0 \mu_{0} 1$ & $r$ & $\circ$ & \\
\hline 0,0099 & - PADT & 0,0097 & 0,0194 & $0 / \circ r V$ & $0,0 Y 19$ & $r$ & & \\
\hline $0 / 0110$ & $\circ / 0 \wedge 9 V$ & $0 / 0 \Delta Y V$ & $0,0 \mid M Y$ & $0,091 \mathrm{~V}$ & 0,0991 & 1 & & \\
\hline 0,0094 & $0, \pi 111$ & 0,0 pro & $0,0|V|$ & O.० TVA & 0,0 prg & $r$ & 1 & $\lambda$ \\
\hline$\circ, \circ \circ \wedge \mathrm{V}$ & optir & 0,0 YKN & 0,0191 & ०० VGY & $0,0 \mathrm{FVT}$ & $r$ & & \\
\hline$\circ, 0 \circ \wedge 9$ & $\circ, 0 \wedge \Delta r$ & $0,14 \|$ & 0,0194 & $-0,0 \circ \mu$ & $0,090 r$ & 1 & & \\
\hline$\circ, 0 \circ \wedge r$ & $0, \pi Y q T$ & $0, \pi Y \wedge 1$ & 0,0 Y D & $0,0 Y 09$ & צח & $r$ & $r$ & \\
\hline$\circ, 0 \circ \wedge \mu$ & $0, \uparrow \wedge \circ 1$ & $0 / \Delta G Y Y$ & $0,0 Y \mid \Lambda$ & $\circ, 0 \wedge 91$ & 0,1999 & $r$ & & \\
\hline
\end{tabular}

امر ارتباط مستقيم حجم نمونه با مقدار p است. با افزايش مقدار p، شانس برطرف شدن خرابى با تعمير 
مينيمال كاهش يافته و لذا تعداد خرابىهاى كمترى مشاهده مىشود. براى بررسى توان آمارههاى آزمون

جدول Y. اريبى و ميانكين توانهاى دوم خطاى بارامترهاى مدل r.

\begin{tabular}{|c|c|c|c|c|c|c|c|c|}
\hline \multicolumn{3}{|c|}{ ميانخين توانهاى دوم خطا } & \multicolumn{3}{|c|}{ اريبى } & \multirow{2}{*}{$\lambda$} & \multirow{2}{*}{$\alpha$} & \multirow{2}{*}{$\mathrm{p}$} \\
\hline$\hat{p}$ & $\hat{\lambda}$ & $\hat{\alpha}$ & $\hat{p}$ & $\hat{\lambda}$ & $\hat{\alpha}$ & & & \\
\hline $0,00 \Delta Y$ & 0,1194 & $0,01 V 9$ & 0,0049 & $0, \pi \backslash \Delta \Delta$ & $0,0 M Y I$ & 1 & & \\
\hline $0,0 \circ T V$ & oगVKr & 0,00911 & $0,00 k 4$ & $0,0 V \circ 9$ & 0,0 YIS & $r$ & ه & \\
\hline $0,00 \mu T$ & $0, \mu \circ 10$ & $0,0 \circ V^{t}$ & $0,004_{0}$ & $0,0 \mathrm{YDV}$ & $0,0 \mathrm{MA}$ & $r$ & & \\
\hline $0,0 \circ \varphi_{0}$ & $\circ, 0 V V A$ & 0,0 kys & 0,0040 & o,otro & $0,04 \lambda 1$ & 1 & & \\
\hline $0,00 \mu$ & $0,19 \wedge 9$ & $0,0 \mathrm{TVV}$ & $0,00 k 4$ & $0,00 \psi_{0}$ & $0,0 \mathrm{HVI}$ & r & 1 & $0 / 1$ \\
\hline $0,00 Y 9$ & $0, \pi 009$ & 0,0 TOD & $0,004 \pi$ & $0,0 \mu \pi 0$ & $0,0<00$ & $r$ & & \\
\hline $0,0 \circ Y 9$ & $0,0 V M r$ & $0 / 1011$ & 0,0040 & $-0,0 \| 1 k$ & $0,0 \wedge 11$ & 1 & & \\
\hline $0,00 Y 9$ & 0,1991 & 0,0991 & $0,00 \mathrm{kT}$ & $-0,001 Y$ & $0,0 \wedge 90$ & r & $r$ & \\
\hline $0,00 Y 9$ & $0, \pi \circ 19$ & 0,0991 & 0,0040 & 0,0491 & $\circ, 0 \wedge V \Delta$ & $r$ & & \\
\hline $0 / 0101$ & 0,1 HAF & 0,0190 & $0,0 \circ V \mu$ & 0, TYIT & 0,0 KYA & 1 & & \\
\hline$\circ, 0 \circ V 9$ & $0, r_{0} 11$ & 0,0110 & 0,0019 & س & 0,0 TGY & r & $\circ$ & \\
\hline $0,009 V$ & $0, \mu \Lambda \backslash \Lambda$ & $\circ, 0 \circ \wedge \Delta$ & $\circ, 0 \circ \wedge \Lambda$ & 0,0119 & $0,0 Y M$ & $r$ & & \\
\hline $0,0 \circ \wedge 0$ & $0,0 \wedge \mu K$ & 0,0490 & $0,00 \wedge T$ & 0,0DTr & $0,0 \Delta V \Delta$ & 1 & & \\
\hline 0,0090 & 0,1914 & O THD & $0,00 \wedge 9$ & $0,0 Y 01$ & 0,0 pro & r & 1 & \\
\hline 0,0094 & - TrVTD & $0,0 H \circ V$ & $0,0 \circ \wedge \Lambda$ & 0,0900 & $0,0 \vee M \Lambda$ & $r$ & & \\
\hline $0,009 \pi$ & $0,0 \vee 91$ & ONKT & 0,0090 & $-0,0 \circ \mathrm{VA}$ & $0,099 V$ & 1 & & \\
\hline 0,0094 & $0,194 \pi$ & OMTIK & 0,0090 & $0,01 T \Lambda$ & 0,1049 & r & $r$ & \\
\hline 0,0094 & orrVTr & 0,1419 & 0,0097 & 0,0991 & $0,10 Y T$ & $r$ & & \\
\hline $0,0|4|$ & $0,19 \Delta Y$ & 0,0 TO9 & 0,0100 & $0, T V Y i$ & 0,0DTr & 1 & & \\
\hline 0,0111 & OKYTV & $0,01 K \Lambda$ & $0,01 Y V$ & $0 / 1 \mu_{0}$ & 0,0 MT & $r$ & $\circ$ & \\
\hline $0,010 Y$ & $0 / 01$ G & 0,0101 & 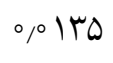 & هTrות & $0,0 Y 91$ & $r$ & & \\
\hline 0,0111 & 0,0900 & $0,0 \Delta 009$ & $0,01 Y T$ & 0,0949 & 0,0991 & 1 & & \\
\hline 0,0101 & O MTOA & 0,0490 & טמו & $0,0 Y M$ & $0,0 \Delta r G$ & $r$ & 1 & \\
\hline 0,0091 & $.01 Y V$ & 0,0494 & $0,01 \mathrm{KT}$ & $0 / 1 \mathrm{VT}$ & $0,0 \Delta \Lambda T$ & $r$ & & \\
\hline $0,009 V$ & $0,0 \wedge \Delta \wedge$ & $0 / \Delta P q$ & 0,0140 & $-0,0009$ & $0,11 \wedge 9$ & 1 & & \\
\hline 0,0099 & orTाו & $0 / \Delta \Delta r$ & טגו" & 0,0 HGV & 0 OTYYV & r & $r$ & \\
\hline $0,009 V$ & $\circ \Delta \circ \Delta r$ & 0 गDVG & $0,01 \mu x$ & $0 \lambda Y \circ Y$ & 0 oltrg & $r$ & & \\
\hline
\end{tabular}




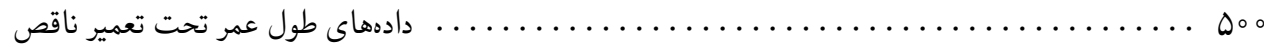

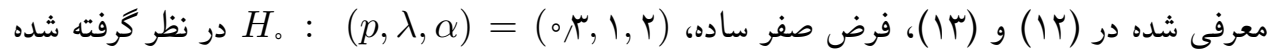

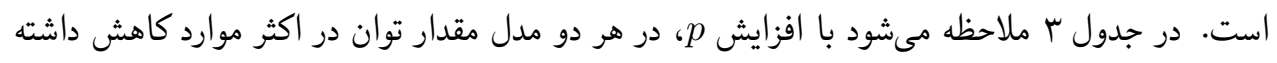

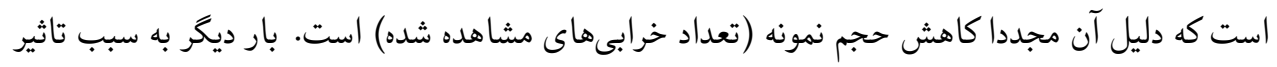

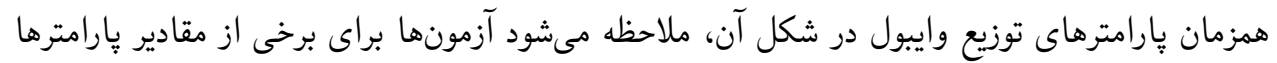

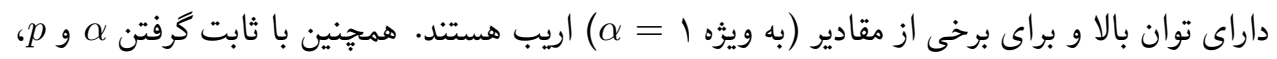

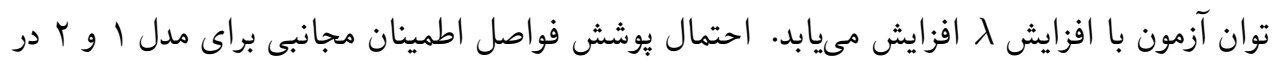

\begin{tabular}{|c|c|c|c|c|c|c|c|}
\hline \multicolumn{6}{|c|}{$p$} & \multirow[b]{3}{*}{$\lambda$} & \multirow[b]{3}{*}{$\alpha$} \\
\hline \multicolumn{2}{|c|}{0} & \multicolumn{2}{|c|}{$0, r$} & \multicolumn{2}{|c|}{0,1} & & \\
\hline مدل r & مدل 1 & مدل r & مدل 1 & مدل r & مدل 1 & & \\
\hline 0,0 rDI & $0,01 Y V$ & $0,0 \mathrm{MVV}$ & 0,0119 & $0,049 \Lambda$ & 0,01149 & 1 & \\
\hline $0, \mu r q 4$ & $0,19 V r$ & $\circ \mu \Lambda \circ V$ & OKTOK & oxpyo & - TOIV & $r$ & 0 \\
\hline$\circ \wedge Y \circ V$ & $0,9 \mathrm{VVI}$ & - $\wedge 994$ & ONTYV & $0,9 \circ \wedge 1$ & $\circ \mathrm{NN1}$ & $r$ & \\
\hline $0,0 \circ 0 \psi^{k}$ & \%,000\% & $0,000 \wedge$ & $0,000 k^{c}$ & $0,000 \wedge$ & 0,0004 & 1 & \\
\hline $0,0 \circ V_{0}$ & 0,0010 & $0,0|V|$ & 0,0010 & $0,0 \Delta F^{\prime}$ & $0,0 \circ \wedge \circ$ & $r$ & 1 \\
\hline 0,1909 & $\circ, 0 \mathrm{VDI}$ & o r rVA & 0,1990 & $0,94 \wedge 9$ & 0,4949 & $r$ & \\
\hline$*$ & $*$ & $0 / Y_{0} \wedge$ & $0, Y \circ \mid Y$ & $0, \mu \mid V$ & ת מ & 1 & \\
\hline 0,0 TYq & $0,0 \wedge 1 \wedge$ & $0, \lambda V x_{0}$ & $0, x q \vee q$ & $0,9 \wedge I V$ & $0,919 V$ & $r$ & $r$ \\
\hline 0,1091 & $0, \pi \mid r \Delta$ & 0 preqv & $\circ V I V A$ & $\circ, 9 \circ \Delta \Delta$ & 0,9941 & $r$ & \\
\hline
\end{tabular}

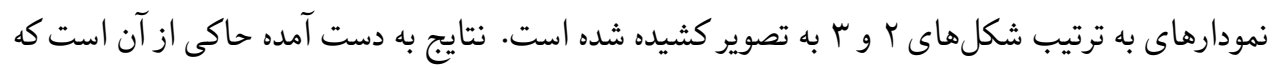

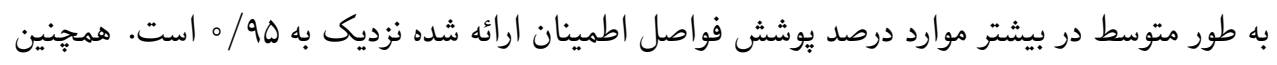

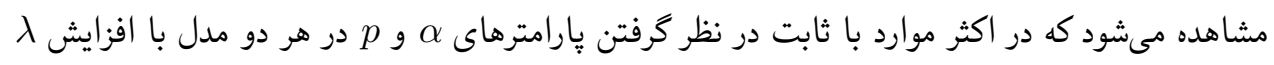

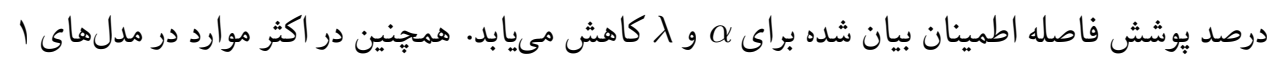

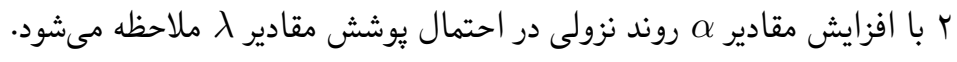



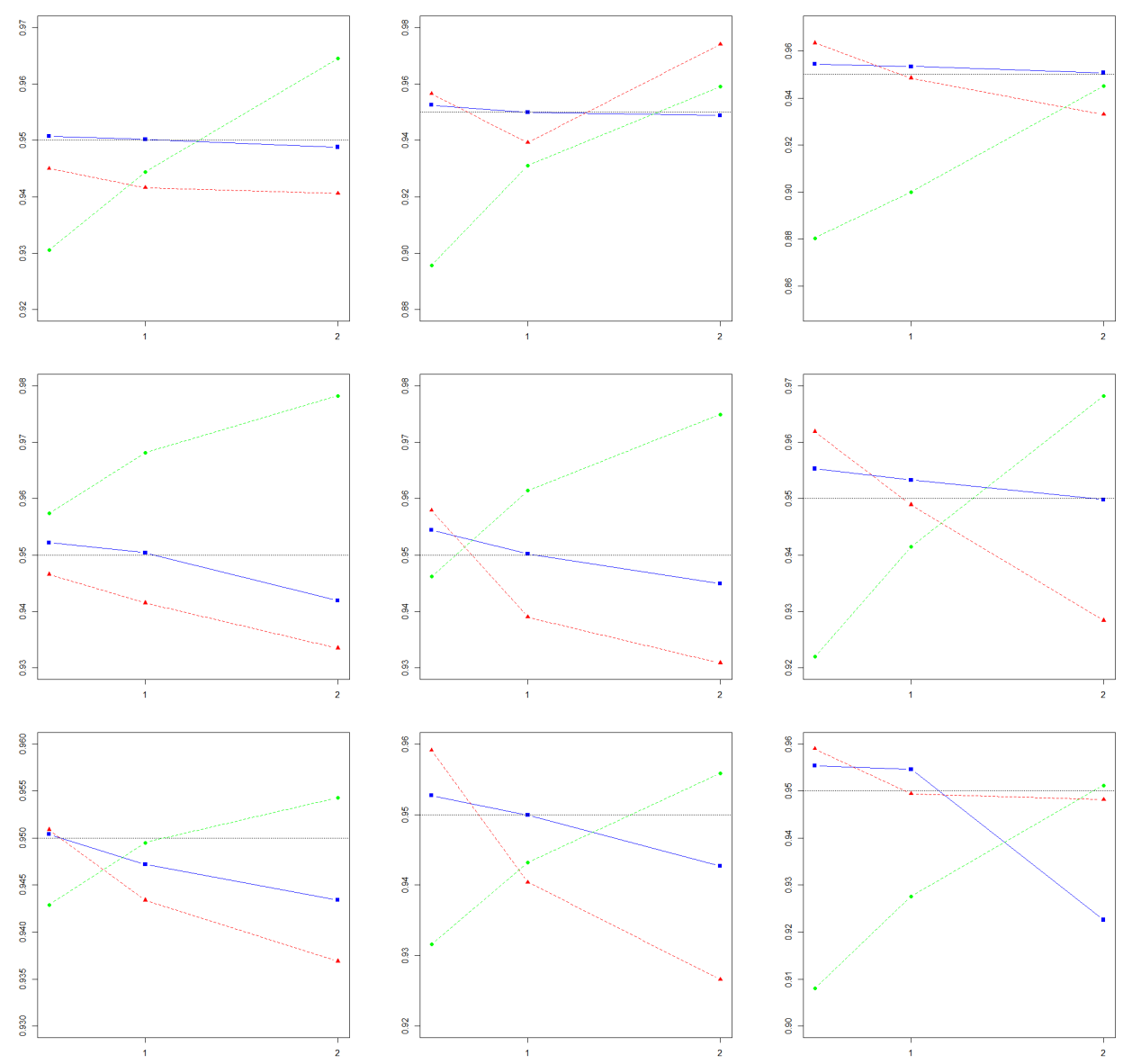

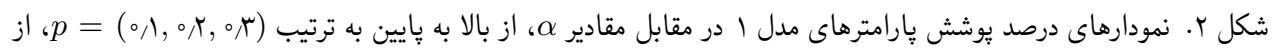

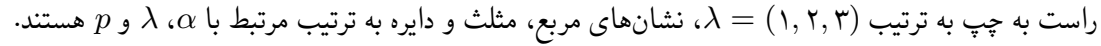

\section{بحث و نتيجهگیرى}

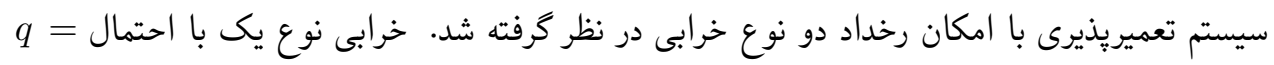

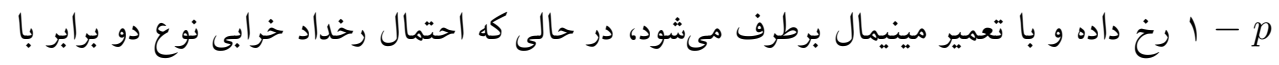

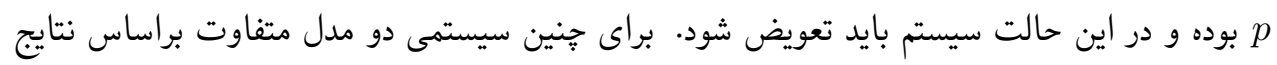

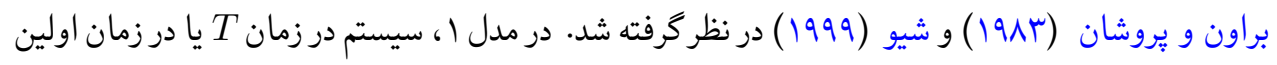

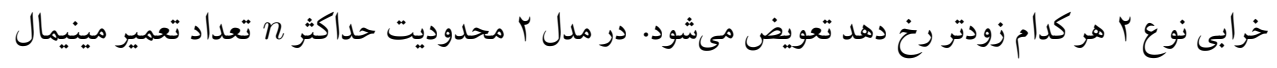



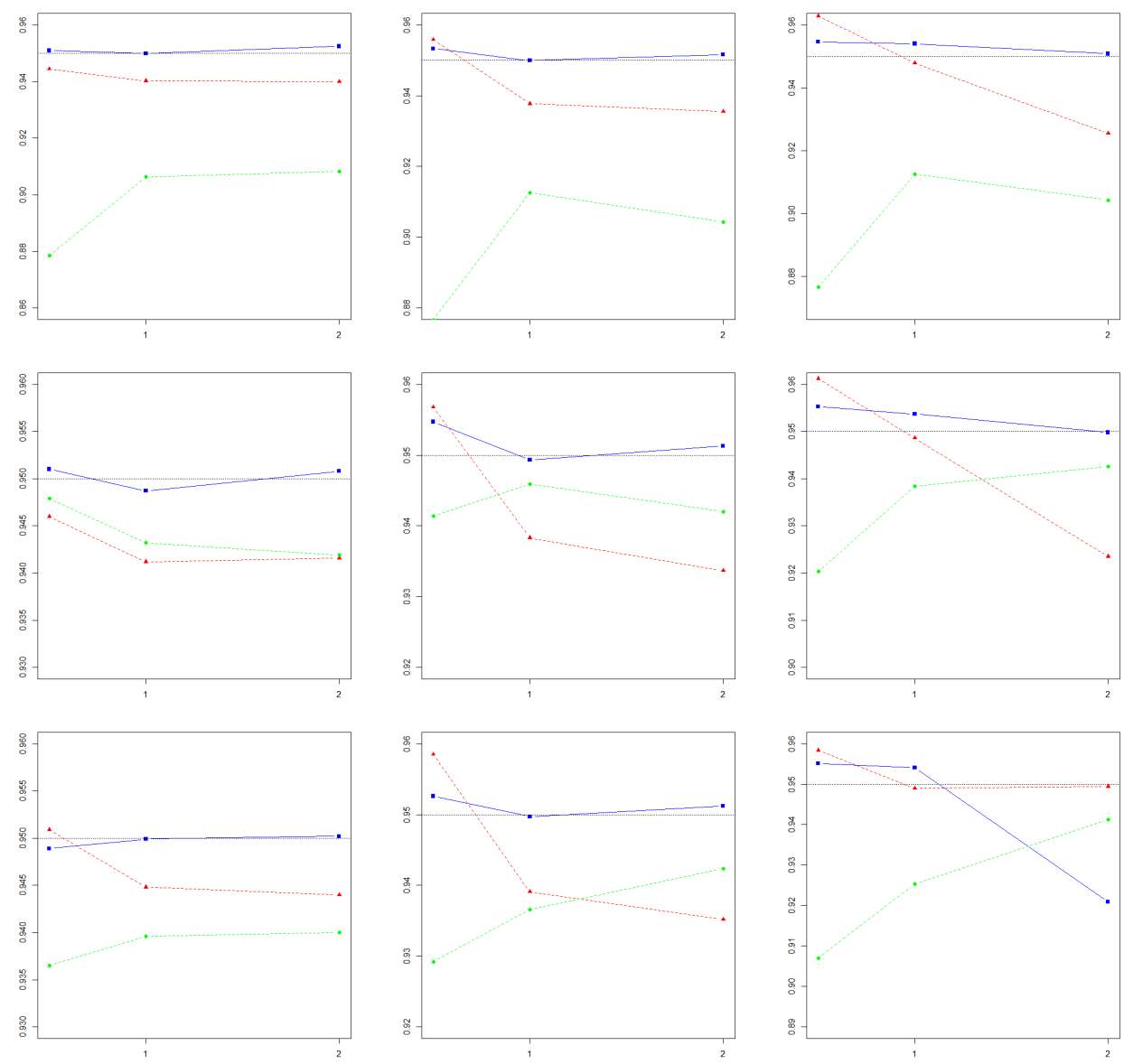

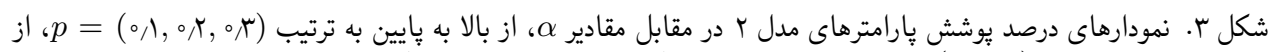

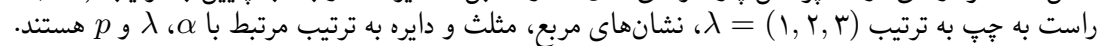

نيز اضافه مىشود. با در نظر گرفتن توزيع وايبول به عنوان توزيع طول عمر اوليه سيستم، الكوريتمهايى براى

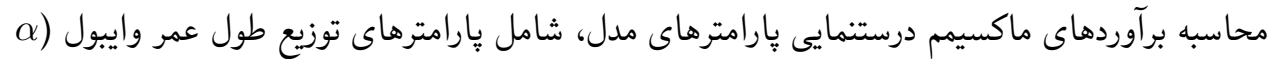

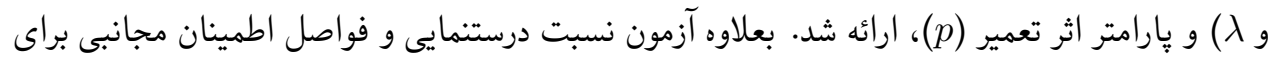

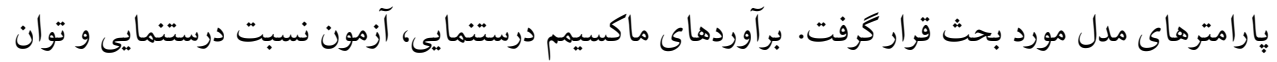

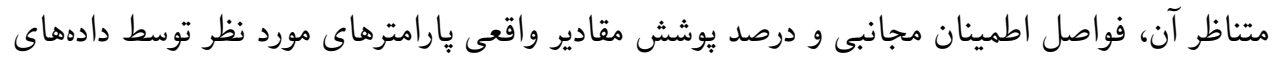

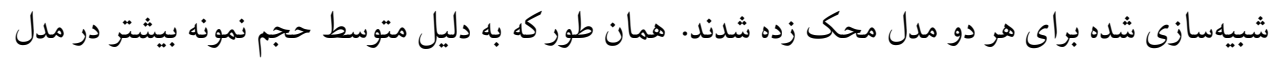




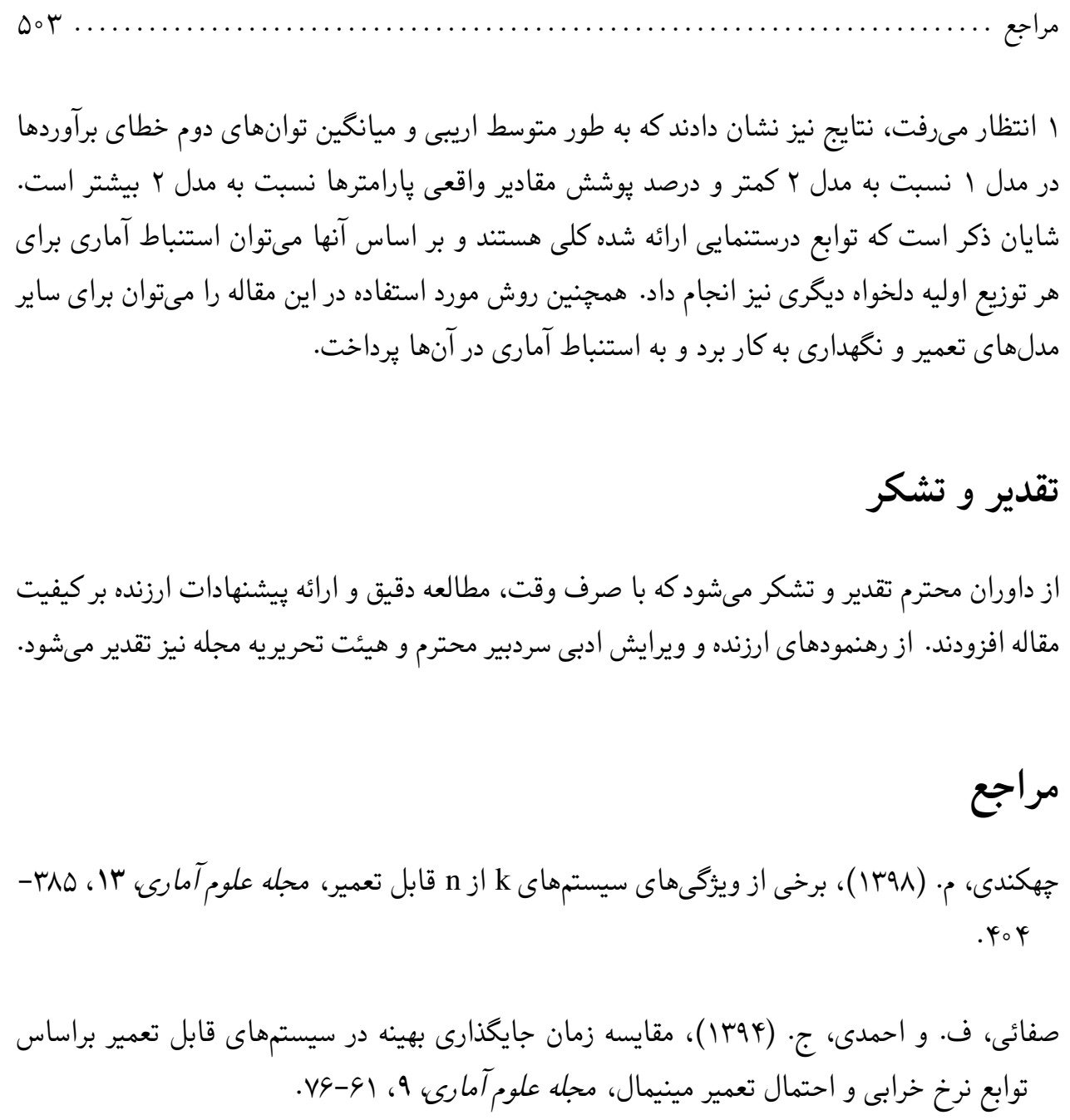

Block, H. W., Borges, W. S. and Savits, T. H. (1985), Age-Dependent Minimal Repair, Journal of Applied Probability, 22, 370-385.

Block, H. W., Borges, W. S. and Savits, T. H. (1988), A General Age Replacement Model with Minimal Repair, Naual Research Logistics, 35, 365-372.

Brown, M. and Proschan, F. (1983), Imperfect Repair, Journal of Applied Probability, 20, 851-859. 
Cheng, G. Q. and Li, L. (2012), A Geometric Process Repair Model with Inspections and its Optimization, International Journal of Systems Science, 43, 1650-1655.

Sheu, S. H. (1999), Extended Optimal Replacement Model for Deteriorating Systems, European Journal of Operational Research, 112, 503-516.

Sheu, S. H., Chen, Y. L., Chang, C. C. and Zhang, Z. G. (2012), A Block Replacement Policy for Systems Subject to Non-Homogeneous Pure Birth Shocks, IEEE Transactions on Reliability, 61, 741-748.

Sheu, S. H., Chen, Y. L., Chang, C. C. and Zhang, Z. G. (2013), Optimal Number of Repairs Before Replacement for a System Subject to Shocks of a Non-Homogeneous Pure Birth Process, IEEE Transactions on Reliability, 62, 73-81.

Sheu, S. H., Chen, Y. L., Chang, C. C. and Zhang, Z. G. (2016), A Note on a Two Variable Block Replacement Policy for a System Subject to NonHomogeneous Pure Birth Shocks, Applied Mathematical Modelling, 40, $3703-3712$.

Sheu, S. H. and Griffith, W. S. (1996), Optimal Number of Minimal Repairs before Replacement of a System Subject to Shocks, Naval Research Logistics (NRL), 43, 319-333.

Tsai, H. N., Sheu, S. H. and Zhang, Z. G. (2017), A Trivariate Optimal Replacement Policy for a Deteriorating System Based on Cumulative Damage and Inspections, Reliability Engineering and System Safety, 160, 74-88. 
Journal of Statistical Sciences, Autumn and Winter, 2020

Vol. 14, No. 2, pp 487-504

DOI: $10.29252 /$ jss.13.2.487

\title{
The Analysis of Weibull Lifetime Data Subject to Imperfect Repair
}

\section{Kamranfar، H.، Etminan، J. and Chahkandi، M.}

Department of Statistics, University of Birjand, Birjand, Iran.

\begin{abstract}
A repairable system with two types of failures is studied. Type I failure (minor failure) is removed by a minimal repair, whereas type II failure (catastrophic failure) is modified by an unplanned replacement. The first failure of the system follows a Weibull probability distribution and two maintenance policies are considered. In the first policy, the system is replaced at time $T$ or the first type II failure, and in the second policy, the system is replaced at the $n$th type I failure, the first type II failure or at time $T$, whichever takes place first. This paper aims to derive a general representation for the likelihood function of the proposed models. The likelihood-ratio test statistic, maximum likelihood estimators and asymptotic confidence intervals for the parameters are also found. Finally, a Monte Carlo simulation is conducted to illustrate the results.
\end{abstract}

Keywords: Statistical inference, Imperfect repair, Repairable system, Monte Carlo simulation.

Mathematics Subject Classification (2010): 90B25, 62N02. 